\title{
Internethandel mit Lebensmitteln
}

\author{
Nico Ruf
}

Online publiziert: 26 July 2011

(C) Bundesamt für Verbraucherschutz und Lebensmittelsicherheit (BVL) 2011

Für den Großteil der Menschen in der Bundesrepublik Deutschland gehört das Internet mit seinen zahllosen Möglichkeiten und Angeboten längst zum Lebensalltag. Dazu zählt auch der Online-Einkauf. Während in der EU im Jahr 2008 durchschnittlich $24 \%$ der Bevölkerung Waren online bestellten, war der Anteil der Internet-Käufer hierzulande mit $42 \%$ fast doppelt so hoch. Damit liegen die Deutschen im EU-Vergleich hinter Großbritannien (49\%), Dänemark (47\%) und den Niederlanden (43\%) auf dem vierten Rang (BITKOM 2009). Auch die OnlineEinkaufsmöglichkeiten für Lebensmittel sind mittlerweile vielfältig, die Anbieter oftmals jedoch regional orientiert und nicht einfach zu finden. Die großen Supermarktketten und Händler sind bis auf wenige Ausnahmen bislang mit ihren Angeboten noch sehr zurückhaltend. Demgegenüber bestätigen Umfragen ein hohes Interesse der Verbraucher an Online-Angeboten. ${ }^{1}$ Gegenwärtig werden häufig Delikatessen im Internet bestellt, die im lokalen Handel nur schwer zu bekommen sind. Zudem werden oft Tiefkühlkost und Weine erworben, während frische Produkte meist nur in großen Ballungsräumen wie Berlin oder München online angeboten werden. Insgesamt wurden im Jahr 2006 Lebensmittel und Getränke im Wert von etwa 122 Millionen Euro im Internet bestellt (BITKOM 2007). Bei einem Gesamtumsatz im Lebensmittelhandel von 138,2 Milliarden Euro entfielen damit weniger als 0,1\% auf

\section{N. Ruf (ه)}

Bundesamt für Verbraucherschutz und

Lebensmittelsicherheit, Mauerstr. 39-42, 10117 Berlin,

Germany

e-mail: nico.ruf@bvl.bund.de
Online-Einkäufe (Magazin für Verbraucher 2007). 2009 lag der Anteil bereits bei 0,5\% (E-Commerce Magazin 2011). Trotz dieser Umsatzsteigerung steht die Entwicklung des Online-Lebensmittelhandels in Deutschland erst am Anfang.

Manche EU-Länder sind hier schon viel weiter. So wurden in Großbritannien im Jahr 2009 Lebensmittel im Wert von fast 6 Milliarden Pfund Sterling, was etwa 7,2 Mrd Euro entspricht, online bestellt (Leggatt 2010). Die großen britischen Handelsketten wie Sainsbury's, Tesco, Asda und Waitrose bieten ihr gesamtes Sortiment landesweit auch online an. Das Internet wird sehr erfolgreich als zusätzlicher Vertriebskanal genutzt (Krewinkel et al. 2011), und die bestellten Waren aus dem nahegelegenen Supermarkt geliefert. Daher verwundert es nicht, dass sich deutsche Handelsketten mittlerweile personelle Unterstützung aus dem Vereinigten Königreich holen, um den eigenen Online-Service aufzubauen (Krisch 2010).

Aus Verbrauchersicht ist diese Entwicklung durchaus positiv zu bewerten. Immerhin bietet der Online-Lebensmitteleinkauf viele Vorteile. Zunächst ist man nicht an Öffnungszeiten gebunden; man muss sich nicht in die obligatorische Schlange an der Kasse einreihen und das Gekaufte nicht in die eigene Wohnung tragen. Wer keinen Supermarkt vor Ort hat, braucht nicht mit dem eigenen Auto in die nächste Stadt zu fahren, sondern bestellt einfach online. Dies könnte sogar positive Auswirkungen auf die Umweltbilanz von Lebensmitteln haben. Denn mehr als 80 \% der Endverbraucher nutzen für ihren

\footnotetext{
${ }^{1}$ Studie „Lebensmitteleinkauf 2011 - Lust oder Frust?“ des Elite News Instituts im Auftrag des Online-Supermarktes froodies.
} 
Weg zum Supermarkt den privaten PKW, was im Durchschnitt etwa 30-40 \% der gesamten Umweltbilanz von Lebensmitteln ausmacht (Schlich 2011). Der wöchentliche Einkauf mit dem Auto kostet also nicht nur Zeit und Geld, sondern verschlechtert die Umweltbilanz von Lebensmitteln erheblich und schadet der Umwelt. Ein gut organisierter Lieferservice könnte hier ökonomisch und ökologisch durchaus sinnvoll sein.

Der Internethandel ganz allgemein und der Online-Handel mit Lebensmitteln insbesondere bergen nicht nur Vor-, sondern auch Nachteile und teilweise Risiken für den Verbraucher. Denn Zeitgewinn und Bequemlichkeit haben ihren Preis. Da sind zunächst die Lieferkosten, die jedoch meist ab einem bestimmten Einkaufswert entfallen. Vermutlich etwas weniger im Bewusstsein der Verbraucher verankert sind Einschränkungen beim Rückgabe- bzw. Widerrufsrecht, da man beispielsweise leicht verderbliche Ware nicht zurückgeben kann. Hinzu kommt das Risiko, dass Ware beim Versand beschädigt werden kann. Wenn der Kunde dies bei der Anlieferung nicht feststellt und die beschädigte Ware annimmt, ist die Zeitersparnis alleine durch die spätere Reklamation wieder aufgehoben. Weiteres lesen Sie dazu im Beitrag von Sophie Mecchia in dieser Ausgabe (Mecchia 2011).

Neben den rechtlichen bzw. wirtschaftlichen Aspekten des Verbraucherschutzes haben die jüngsten Ereignisse um Dioxine und EHEC den gesundheitlichen Verbraucherschutz in den Fokus des öffentlichen Interesses gerückt. Wie sicher sind unsere Lebensmittel? Die von den Bundesländern an das Bundesamt für Verbraucherschutz und Lebensmittel-sicherheit (BVL) übermittelten Daten belegen: Die im Rahmen der amtlichen Überwachung untersuchten Lebensmittel sind überwiegend sicher (Berichte zur Lebensmittelsicherheit 2005 bis 2009). Lebensmittel, die nicht den gesetzlichen Anforderungen entsprechen, werden aus dem Verkehr gezogen. Können diese Aussagen aber auch auf den Online-Handel übertragen werden? Leider nicht, wie drei weitere Beiträge in dieser Ausgabe belegen (Büchter et al. 2011; Löbell-Behrends et al. 2011; Krewinkel et al. 2011). Die Problemfelder liegen in erster Linie in Regelungslücken und in der Anwendung bzw. der Machbarkeit von amtlichen Kontrollen. Dabei ist nicht jedem Online-Händler bekannt, dass es eine Pflicht zur Registrierung gibt. Wen die Behörden nicht kennen, können sie demzufolge auch nicht kontrollieren. Auch durch die wachsenden Umsätze und Angebote von risikoreichen Produkten steigen die Anforderungen an die amtliche Überwachung. Risiken gibt es insbesondere bei den sogenannten Borderline-Produkten. Dabei handelt es sich um Produkte, deren Zuordnung zu Produktgruppen, die meist verschiedenen Regelungen unterliegen, schwierig ist. Dazu zählen beispielsweise Nahrungsergänzungsmittel (NEM), Kosmetika, Bedarfsgegenstände und Arzneimittel. NEM gelten als Lebensmittel, müssen beim BVL registriert werden und unterliegen der amtlichen Lebensmittelüberwachung. Anders verhält es sich bei Arzneimitteln, die durch das Bundesamt für Arzneimittel und Medizinprodukte überprüft und zugelassen werden müssen (Löbell-Behrends et al. 2011). Was kauft der Kunde also, wenn er einen mit Johanneskraut und Melisse angereicherten Joghurt kauft, der beruhigend und ausgleichend wirken soll: ein Lebens- oder ein Arzneimittel? Wenn es zusätzlich zu dieser Problematik noch eine lückenhafte Kennzeichnung der Lebensmittel gibt, weil die geltende Lebensmittelkennzeichnungsverordnung dem Handel nicht vorschreibt, dass im Internet Angaben zu den Zutaten, Allergenen oder Unverträglichkeiten zu machen sind, geht der Verbraucher ein nicht unbeträchtliches Risiko mit seinem OnlineKauf ein. Diese und andere Probleme sind von Fachleuten bereits benannt. Lösungen werden entwickelt oder schon erprobt. Laufende Projekte zur amtlichen Kontrolle des Online-Handels mit Lebensmitteln (Büchter et al. 2011) sowie zur Aufklärung von Verbrauchern (Mecchia 2011) stellen vielversprechende Ansätze dar.

Trotz der Risiken liegt im Online-Handel mit Lebensmitteln ein großes Entwicklungspotential. Man kann davon ausgehen, dass sich dieser zusätzliche Vertriebskanal weiter entwickeln wird. Wie bei allen Transaktionen im Internet obliegt uns Verbrauchern dabei eine Sorgfaltspflicht und ein gewisses $\mathrm{Ma}$ an Eigenverantwortung. Der Handel trägt eine besondere Verantwortung dem Kunden gegenüber und ist verpflichtet, sich an die bestehenden Regelungen zu halten. Der Gesetzgeber und die zuständigen Behörden sind dabei gefordert, rechtsfreie Räume zu verhindern und den Verbraucherschutz zu gewährleisten.

\section{Literatur}

Berichte zu Lebensmittelsicherheit der Jahre 2005 bis 2009. Bundesamt für Verbraucherschutz und Lebensmittelsicherheit. http://www.bvl.bund.de/DE/08_PresseInfothek/04 Publikationen/03_Berichte/infothek_berichte_node.html\# doc1401838bodyText1 
BITKOM (2007) Presseinformation: Deutsche bestellen Lebensmittel für 122 Millionen Euro im Internet. http://www. bitkom.org/files/documents/BITKOM_Presseinfo_OnlineLebensmittel_17.01.2007.pdf

BITKOM (2009) Presseinformation: online-shopping in Deutschland weit verbreitet. http://www.bitkom.org/files/ documents/BITKOM_Presseinfo_E-Commerce_21_05_2009. pdf

Büchter B, Kuhr C, Schreiber GA (2011) Pilotprojekt zur amtlichen Kontrolle des Handels mit Lebensmitteln im Internet. J Verbr Lebensm. doi:10.1007/s00003-011-0700-9

E-Commerce Magazin (2011) Studie: Verbraucher bereit für Internet-Lebensmittelmärkte. http://www.e-commercemagazin.de/ecm/news/studie-verbraucher-bereit-fuerinternet-lebensmittelmaerkte. Zugriff am 04.07.2011

Krewinkel A, Tolg B, Fritsche J (2011) Online-Lebensmittelhandel und Strategien zur Kontrolle des virtuellen Lebensmittelmarktes. J Verbr Lebensm. doi:10.1007/s00003011-0701-8

Krisch J (2010) http://www.excitingcommerce.de/2010/12/kaiserstengelmann-holt-tesco-mann-f\%C3\%BCrs-online-gesch\% C3\%A4ft.html
Leggatt H (2010) BizReport: Ecommerce: January 20, 2010. http:// www.bizreport.com/2010/01/food_for_thought_-_british_ online_grocery_sales_to_soar.html\#

Löbell-Behrends S, Böse W, Maixner S, Kratz E, Kohl-Himmelseher M, Marx G, Lachenmeier DW (2011) Kontrolle des Internethandels mit Lebensmitteln - Abgrenzung bei Borderline-Produkten und Ansätze für effektive Kontrollstrategien. J Verbr Lebensm 6. doi:10.1007/s00003-011-0687-2

Magazin für Verbraucher (2007) vom Presse- und Informationsamt der Bundesregierung Nr. 001 11/2007 Lebensmittel: Produktion und Handel in Deutschland. http://www. bundesregierung.de/Content/DE/Magazine/MagazinVerbrau cher/001/s2-lebenmittelproduktion-und-handel-in-deutschland. html

Mecchia S (2011) Meeresfrüchte per Mausklick: Lebensmittelhandel im Internet. J Verbr Lebensm. doi:10.1007/ s00003-011-0706-3

Schlich E (2011) Umweltbilanz von Lebensmitteln - Sind regionale Lebensmittel immer klimafreundlicher? http:/ www.vdoe.de/fileadmin/redaktion/download/jahrestagung/ 2011/Statement-Schlich.pdf 